\title{
Identification of plasma microRNA profiles for primary resistance to EGFR-TKIs in advanced non-small cell lung cancer (NSCLC) patients with EGFR activating mutation
}

Shuhang Wang ${ }^{1}$, Xiaomei Su${ }^{1}$, Hua Bai ${ }^{1}$, Jun Zhao', Jianchun Duan¹, Tongtong An ${ }^{1}$, Minglei Zhuo ${ }^{1}$, Zhijie Wang ${ }^{1}$, Meina $\mathrm{Wu}^{1}$, Zhenxiang $\mathrm{Li}^{1}$, Jian $\mathrm{Zhu}^{2}$ and Jie Wang ${ }^{1,3^{*}}$

\begin{abstract}
Background: EGFR mutation is a strong predictor of efficacy of epidermal growth factor receptor tyrosine kinase inhibitor (EGFR-TKIs) therapy in advanced non-small cell lung cancer (NSCLC). However, around 20-30\% of EGFR-mutated cases showed no response to EGFR-TKIs, suggesting that other determinants beyond EGFR mutation likely exist. This study analyzed the role of microRNAs (miRNAs) in primary resistance to EGFR-TKIs in advanced NSCLC patients with EGFR mutation.

Methods: Training group: 20 advanced NSCLC patients with EGFR 19 deletion treated with first-line EGFR-TKIs were enrolled; half of them had dramatic responses while the other half had primary resistance. Matched plasma samples were collected for miRNA profiling using TaqMan low-density array (TLDA). Bioinformatics analyses were used to identify related miRNAs possibly accounted for resistance. Testing group: Quantitative reverse transcriptase PCR (qRT-PCR) was employed to detect the level of miRNA with significant differential expression in the training set. Validation group: Another cohort with EGFR 19 deletion mutations, who had dramatically different responses to EGFR-TKI, was used to validate the difference of miRNA expression between the sensitive and resistant groups using RT-PCR.
\end{abstract}

Results: Training group: 153 miRNAs were found to be differentially expressed between the sensitive and resistant groups. Potential target genes were predicted with a target scan database. Twelve differentially expressed miRNAs were selected for the analysis because of their known roles in tumorigenesis of lung cancer, resistance to drugs, and regulation of EGFR pathway. Training group: three out of the 12 miRNAs (miR-21, AmiR-27a, and miR-218) were verified to have significantly higher expression $\left(P_{\text {miR-21 }}=0.004, P_{\text {miR-27a }}=0.009, P_{\text {miR-218 }}=0.041\right.$, respectively $)$ in the resistant group compared to the sensitive group. Validation group: The expression levels of these three miRNAs were validated to be significantly different $(P=0.011,0.011,0.026$, respectively) in the validation cohort $(n=34)$. (Continued on next page)

\footnotetext{
* Correspondence: wangjie_cc@yahoo.com

Shuhang Wang and Xiaomei Su are the first authors.

'The Key Laboratory of Carcinogenesis and Translational Research (Ministry

of Education), Beijing, China

${ }^{3}$ Department of Bioscience and Nutrition, Novum, Karolinska Institute, 14183

Huddinge, Sweden

Full list of author information is available at the end of the article
}

\section{Ciomed Central}

(c) 2015 Wang et al. Open Access This article is distributed under the terms of the Creative Commons Attribution 4.0 International License (http://creativecommons.org/licenses/by/4.0/, which permits unrestricted use, distribution, and reproduction in any medium, provided you give appropriate credit to the original author(s) and the source, provide a link to the Creative Commons license, and indicate if changes were made. The Creative Commons Public Domain Dedication waiver (http://creativecommons.org/publicdomain/zero/1.0/) applies to the data made available in this article, unless otherwise stated. 
(Continued from previous page)

Conclusions: Higher expression levels of miR-21, AmiR-27a, and miR-218 detected in this study suggest potential roles of these miRNAs in primary resistance to EGFR-TKI in advanced NSCLC patients with EGFR exon 19 deletion mutations. These findings need to be further confirmed in a study with a larger sample size.

Keywords: EGFR-TKI, EGFR 19 deletion mutation, microRNA and primary resistance

\section{Background}

Non-small cell lung cancer (NSCLC) is a leading cause of cancer-related death. Systemic chemotherapy remains palliative and modestly effective. Recently, multiple prospective clinical studies have shown that EGFR mutation is a strong predictor of efficacy of epidermal growth factor receptor tyrosine kinase inhibitor (EGFR-TKIs) therapy in advanced NSCLC. More than $70 \%$ of NSCLC patients carrying EGFR mutations achieved marked and durable responses to treatment with the EGFR-TKIs gefinitib or erlotinib [1, 2]. However, although several potential mechanisms of primary resistance, including Bim deletion polymorphism [3], KRAS mutation [4], and ALK fusion [5] have been explored in several preclinical and retrospective studies, the molecular basis of primary resistance to EGFR-TKI remains unclear.

MicroRNA is a newly defined class of small noncoding RNAs of 21-25 nucleotides in length that has recently been implicated in cancer biology, which could posttranscriptionally regulate gene expression by binding to complementary sequences in the 3 ' untranslated region (3'UTR) of the target messenger RNA [6]. This could ultimately lead to repression of protein translation and down-regulation of protein expression [7]. Deregulation of microRNAs (miRNAs) is emerging as an important area of study in carcinogenesis because their regulatory capabilities can drastically influence cell physiology [8], and it was also reported to be with EMT which might be attributed to resistance to anti-tumor therapy $[9,10]$. Many studies have examined miRNA expression profiles with the goal of identifying miRNA using non-invasive blood samples as biomarkers for the diagnosis of lung cancer. Most of these studies have quantified miRNAs in free-cellular cfDNA of serum $[8,11]$ or plasma [12-14], and a new strategy could be investigated accordingly [15]. Although all these studies have shown promising results, there are some limitations with the use of serum or plasma RNA for miRNA biomarker discovery. We proposed that miRNAs might regulate the EGFR gene pathway and could be a predictor of response to EGFRTKI therapy. Genomic loss of miRNAs capable of downregulating EGFR would be expected to enable increased EGFR expression, thereby offering a more robust target for the EGFR-TKIs.

Based on the fact that primary resistance to EGFRTKIs exists in a portion of patients with a sensitizing mutant-type, it is critical to identify potential biomarkers that can help determine the subgroup of patients with primary resistance to EGFR-TKIs therapy. In this study, we investigated the expression profiles of miRNAs in EGFR-mutated NSCLC patients with either dramatic responses or primary resistance to EGFR-TKI therapy, and explored which miRNA may play a role in primary resistance to EGFR-TKIs in EGFR-mutant patients. Finally, we propose strategies to avoid primary resistance to EGFR-TKIs in the near future.

\section{Materials and methods}

Patients enrolled in this study were diagnosed pathologically with NSCLC stage IIIB-IV or recurrent disease and received EGFR-TKIs treatment from April 2004 to August 2012 at the Beijing Cancer Hospital. All of the patients harboring EGFR 19 deletion mutations detected in both plasma and tissues received $250 \mathrm{mg}$ of gefitinib or $150 \mathrm{mg}$ of erlotinib daily until disease progression, intolerable toxicity, or patient refusal. None of the patients had KRAS mutation, T790M mutation, or C-MET amplification. All of the patients had bi-dimensionally measurable disease and presented an Eastern Cooperative Oncology Group (ECOG) performance status of 0 to 2. Blood samples were collected prior to EGFR-TKI treatment for biomarker analysis. All patients provided written informed consent and a separate consent was obtained for the optional provision of a tumor sample for biomarker analysis. The Institutional Ethics Committee at Beijing Cancer Hospital approved the study protocol.

\section{Study design}

The study was designed to explore the potential role of miRNAs in primary resistance to EGFR-TKIs treatment. Tumor specimens were obtained at initial diagnosis. Clinical data were sealed during the laboratory analysis until all data were evaluated. Recorded variables included age, sex, smoking history, pathology, and ECOG performance status, stage at diagnosis, treatments, and toxicities. Outcome indicators included progression-free survival (PFS) and overall survival (OS). We defined primary resistance of EGFR-TKI as PFS $\leq 3$ months (90 days) without any evidence of objective response while receiving EGFR-TKI [16].

Although both EGFR exon 19 deletions and 21 mutations (L858R) are sensitive aberrances which present 
excellent efficacy to EGFR-TKIs treatment, recently, a serial of pooled and meta-analysis comparing first-line chemotherapy in patients with EGFR mutations with first- and second-generation EGFR-TKIs showed that patients with EGFR 19 del had significantly longer overall survival time (OS) or/and PFS compared to those treated with platinum-based chemotherapy. On the contrary, the patients with L858R mutation had longer OS or/and PFS in the chemotherapy group than in EGFRTKIs treatment group [17]. Thus, it has reached the consensus that EGFR19 del and L858R mutations are two different diseases and required different treatment strategies. We therefore speculated that primary resistant mechanism of EGFR exon 19 deletion and L858R mutant may be different. The current study focuses on EGFR 19 deletion mutant NSCLC.

Three cohorts of patients were selected randomly from our database:

Training group: There were 20 advanced NSCLC patients with EGFR 19 deletion treated with first-line EGFR-TKIs, 10 of whom responded dramatically with a PFS over 13 months, while the other 10 presented primary resistance with a relatively short PFS of less than 3 months. Matched plasma was collected for miRNA profile detection using TaqMan low-density array (TLDA).

Testing group: Real-time PCR was used to evaluate the differential expression of miRNAs identified in the training group. Bioinformatics analyses were applied to explore the possible miRNAs that could have been involved in the observed resistance.

Validation group: The set included two cohorts of 17 patients each harboring EGFR 19 deletion mutations, with dramatic responses and primary resistance to EGFR-TKIs in each cohort were used to confirm the miRNAs identified in the training and testing sets. Another cohort of 48 EGFR wild-type patients with dramatic response (24 patients) or primary resistance to EGFR-TKI was chosen to verify reversely that identified miRNAs in the first two groups attributing to primary resistance of EGFR-mutant NSCLC patients have no relation with resistance of EGFR wild-type NSCLC patients.

\section{Assessments}

Tumor assessments, including computed tomography scans of the chest, type-B ultrasonic inspection of bilateral cervical lymph nodes and upper abdomens, magnetic resonance imaging scans of brains, and emission computed tomography scans of bone, were performed at baseline and every 8 weeks until the investigators documented disease progression or unacceptable toxicity. The clinical responses to TKIs including complete response $(\mathrm{CR})$, partial response $(\mathrm{PR})$, stable disease $(\mathrm{SD})$ and disease progression (PD) were determined on the basis of the Response Evaluation Criteria in Solid Tumors (RECIST 1.1) [18]. PFS was defined as the time from the beginning of TKI treatment to PD or death, and OS was defined as the time from the beginning of TKI treatment to death. An independent radiologist (Dr. Ning Wang) assessed all films and was blinded to the EGFR biomarker status.

\section{Specimen collection and DNA extraction}

Each enrolled patient from our database exhibited specific EGFR mutation status (exon 19 deletion) consistently in both tissue and blood. All patients provided sufficient blood samples for miRNA analysis. Prior to TKI or second-line chemotherapy, $4 \mathrm{~mL}$ of anticoagulated venous blood from each patient was collected and placed at $4{ }^{\circ} \mathrm{C}$ for $4 \mathrm{~h}$, then centrifuged at $2500 \mathrm{rpm}$ for $10 \mathrm{~min}$ at low temperature. The plasma was aspirated to a new centrifuge tube, and the high speed centrifugation was repeated. Of the plasma, $1.5 \mathrm{~mL}$ was obtained, to which $15 \mu \mathrm{L}$ of protease $\mathrm{K}(20 \mathrm{mg} /$ $\mathrm{mL}$ ) and 50ro SDS (20 \%) was added to $1.5 \mathrm{ml}$ of plasma. DNA was extracted using phenol/chloroform/isopentanol after a 2-h incubation in a water bath at $60{ }^{\circ} \mathrm{C}$. DNA was precipitated in alcohol and centrifuged the next day. After salt washing, the precipitate was dissolved in TE and then preserved at $-20^{\circ} \mathrm{C}$.

EGFR activating mutation detection by denaturing high-
performance liquid chromatography and amplification re-
fractory mutation system
Specimen collection, DNA extraction, and denaturing Specimen collection, DNA extraction, and denaturing
high-performance liquid chromatography (DHPLC) were performed according to methods described in our previous publication [19]. The amplification refractory mutation system (ARMS), a more sensitive method for detecting EGFR activating mutation, was used to reevaluate the results detected by DHPLC [20].

\section{Quantification of circulating miRNAs from plasma}

Total RNA was extracted from $350 \mu \mathrm{L}$ of plasma using TRIzol LS reagent (Invitrogen) and dissolved in $10 \mu \mathrm{L}$ of DEPC (diethylprocarbonate) water. The quantity and quality of total RNA were determined with the use of a spectrophotometer (GeneQuant pro, GE Healthcare). miRNAs were quantified by quantitative reverse transcriptasepolymerase chain reaction (qRT-PCR) TaqMan miRNA Assays (Applied Biosystems) according to the manufacturer's instructions. Ten nomograms of total RNA were used to synthesize miRNA-specific complementary DNA (cDNA). PCR was performed in duplicates for 40 cycles using an ABI Prism 7900HT Sequence Detection System (Applied Biosystems). The Ct value was defined as the cycle number at which the fluorescence $(\Delta R n)$ exceeded the 
threshold. The threshold was 0.20 , which was defined as the default setting. To evaluate the miRNA expression levels, PCR was carried out in duplicate, and the average of the $\mathrm{Ct}$ values was converted into $2-\mathrm{Ct}$. If the $\mathrm{Ct}$ value could not be determined because the PCR was run for up to 40 cycles and the miRNA expression level was still below the detection limit, the $\mathrm{Ct}$ value was treated as 40 (i.e., miRNA expression level $=2-40$ ).

\section{MicroRNA microarray analysis of plasma and microRNA quantification}

Total RNA was isolated using mirVana miRNA isolation kit (Ambion). For miRNA cDNA synthesis, RNA was reverse transcribed using a miRNA reverse transcription kit (Applied Biosystems) in combination with the stem-loop Megaplex primer pool (Applied Biosystems). TLDA v2.0 (Applied Biosystems) was performed on the $7900 \mathrm{HT}$ realtime PCR system (Applied Biosystems) according to the manufacturer's protocol (764 small RNAs were profiled for each cDNA sample). PCR cycling conditions were as follows: $95{ }^{\circ} \mathrm{C}$ for $10 \mathrm{~min}$ followed by 40 cycles of $95{ }^{\circ} \mathrm{C}$ for $15 \mathrm{~s}$ and $60{ }^{\circ} \mathrm{C}$ for $1 \mathrm{~min}$. Human U6 small RNA was used as an internal control to normalize RNA input. The data were analyzed using SDS v2.3 software. The $\mathrm{Ct}$ value was defined as the fractional cycle number at which the fluorescence passed the fixed threshold. The fold change was calculated using the 2- $\Delta \Delta \mathrm{Ct}$ method and presented as the fold-expression change in tumors and their adjacent normal tissues after normalization to the endogenous control. Significance was considered to be the $p<0.05$ level.

\section{Target gene prediction}

The target genes of the deregulated miRNAs were predicted with TargetScan; conserved and non-conserved targets of miRNAs were identified using TargetScan 3.1. The list of predicted targets was obtained via download from TargetScan (http://www.targetscan.org/).

Ingenuity Pathway Analysis (IPA) 3.0 (Ingenuity Systems 4) was used to analyze the list of predicted miRNAs targets. Filtering was performed to remove duplicates and to remove genes with no annotation in the Ingenuity Pathways Knowledge Base, resulting in a list of 765 network eligible genes. Each gene identifier was mapped to its corresponding gene object in the Ingenuity Pathways Knowledge Base. Networks of genes were then algorithmically generated. GO analysis was also performed using IPA.

\section{Quantitative PCR}

To verify the accuracy of our microarray and TLDA data, we performed single qRT-PCR for representative miRNAs using TaqMan miRNA Assays (Applied Biosystems) according to the manufacturer's instructions. Briefly, total RNA was extracted from plasma using TRIzol Reagent (Invitrogen, Carlsbad, CA, USA) and used to synthesize cDNA with gene-specific primers. Reverse transcriptase reactions contained $100 \mathrm{ng}$ RNA, $50 \mathrm{nmol} / \mathrm{L}$ stem-loop RT primers, $1 \times$ RT buffer, $0.25 \mathrm{mmol} / \mathrm{L}$ each of the dNTPs, $3.33 \mathrm{U} / \mu \mathrm{L}$ MultiScribe reverse transcriptase, and $0.25 \mathrm{U} / \mu \mathrm{L}$ RNase inhibitor. The $15-\mu \mathrm{L}$ reactions were incubated for $30 \mathrm{~min}$ at $16{ }^{\circ} \mathrm{C}, 30 \mathrm{~min}$ at $42{ }^{\circ} \mathrm{C}, 5 \mathrm{~min}$ at $85{ }^{\circ} \mathrm{C}$, and then kept at $4{ }^{\circ} \mathrm{C}$. The cDNA products were used for the subsequent qRT-PCR analysis. The $20-\mu \mathrm{L}$ PCR reaction included $1.33 \mu \mathrm{L}$ RT product, $1 \times$ TaqMan universal PCR master mix, and $1 \mu \mathrm{L}$ primers and probe mix of the TaqMan miRNA assay kit. Reactions were incubated in a 96-well optical plate at $95^{\circ} \mathrm{C}$ for $5 \mathrm{~min}$, followed by $40 \mathrm{cy}$ cles at $95{ }^{\circ} \mathrm{C}$ for $15 \mathrm{~s}$, and $60{ }^{\circ} \mathrm{C}$ for $1 \mathrm{~min}$. PCR reactions were run on a StepOne Plus real-time PCR machine (Applied Biosystems), and the data were analyzed using SDS v2.3 software. Triplicate samples, validated endogenous controls, and interassay controls were used throughout these experiments. miRNA expression levels were calculated using the $2^{-\Delta \Delta C t}$ method. The relative expression of the miRNA of interest corresponded to the $2^{\text {-miR value }}$.

\section{Statistical analyses}

SPSS 16.0 was used to calculate the statistical significance. Wilcoxon tests were applied to compare the differences of expression for miRNA between the different response groups.

\section{Results}

\section{Training process}

Ten couples (20) of patients who met the enrollment criteria were included into the study as the training group. All patients had EGFR mutation both in tissues and in matched blood and lacked KRAS mutation, T790M, ALK fusion, and c-MET amplification. All patients received EGFR-TKI as the first-line therapy and demonstrated dramatic response or primary resistance to EGFR-TKI, including eight and six women, respectively, nine and six patients with lung adenocarcinomas, respectively, and non-smokers ( $n=7$ in both cases) with median PFS of 21 months and 1.8 months, respectively. The patients' clinicopathological characteristics are listed in Table 1.

A total of 764 miRNAs were profiled for each cDNA sample with TLDA. Using a twofold difference in expression as the cut-off, 20 miRNAs were found to be highly expressed in the group with dramatic response, and 133 miRNAs were highly expressed in the group with primary resistance.

\section{Gene ontology and Kyoto Encyclopedia of Genes and Genomes (KEGG) pathway analysis of the deregulated miRNAs}

To identify the candidates and investigate the cellular function, the signaling pathway and gene ontologies (GOs) of the target genes were analyzed. The results 
Table 1 Clinical characteristics of the Training group

\begin{tabular}{lll}
\hline & Responsive & Resistant \\
\hline Gender & 2 & 4 \\
Male & 8 & 6 \\
Female & 56 & 61 \\
Age & & \\
Median & 3 & 3 \\
Smoking & 7 & 7 \\
Yes & & \\
No & 9 & 6 \\
Pathology & 1 & 3 \\
Ade & 0 & 1 \\
Non-Ade & & \\
Undefined NSCLC & 2 & 3 \\
Stage & 8 & 7 \\
IIIB & 21 & 1.8 \\
IV & &
\end{tabular}

revealed a wide variety of miRNA involved in several signaling pathways (Fig. 1a, b), such as cancer, apoptosis, and EGFR signaling pathway. The miRNA-mRNA interaction network analysis integrated these miRNAs and GO terms by outlining the interactions of miRNA and GO-related genes (Fig. 2a). The targets of the 12 deregulated miRNAs (Table 2) were predicted by TargetScan. miR-448 and miR-605 were upregulated in the sensitive group, whereas miR-628-5p, miR-561, miR-520f, miR409-3p, has-miR-138, miR-296-5p, miR-218,miR-1274B, miR-21, and miR-27a were upregulated in the resistance group.

\section{Testing process}

We tested the same miRNAs identified in the training group (U6 [reference miR], miR-628-5p, miR-561, miR520f, miR-409-3p, miR-138p, miR-296-5p, miR-218, miR1274B, miR-21, miR-27a, miR-488, and miR-605) by using qRT-PCR according to the manufacturer's instructions. miR-21, miR-27a, and miR-218 were found to have significant differential expressions in the plasma of the sensitive and resistance groups.

Table 2 Significantly differentially expressed miRNAs in the plasma of responsive and resistance groups

\begin{tabular}{llll}
\hline miRNA & Responsive group & Primary resistance group & P-value $^{\text {a }}$ \\
\hline miR-21 & $116.50 \pm 42.55$ & $524.38 \pm 58.27$ & 0 \\
miR-27a & $16.16 \pm 8.09$ & $42.03 \pm 13.18$ & 0.01 \\
miR-218 & $0.96 \pm 0.93$ & $2.33 \pm 1.40$ & 0.04 \\
\hline
\end{tabular}

Wilcoxon test

\section{Validation process}

RNAs from another 17 couples (34) of patients harboring EGFR 19 deletion with dramatic response or primary resistance to EGFR-TKIs therapy were evaluated by qRT-PCR analysis for the detection of miR-21, miR-27a, and miR-218. The results are presented in Table 3.

\section{EGFR wild-type group}

miR-21, miR-218, and miR-27a were also detected by qRT-PCR from 24 couples (48) of patients with EGFR wild-type who presented response $(n=24)$ or resistance $(n=24)$ to EGFR-TKIs. Patient characteristics are shown in Table 4, and no significant difference was found.

\section{Discussion}

The mechanism of primary resistance to EGFR-TKIs in EGFR-mutant NSCLC is not clearly understood. In this study, we analyzed miRNA expression signatures related to primary resistance to EGFR-TKIs in EGFR-mutant NSCLC patients. Our results indicate the existence of miRNA expression patterns that could distinguish between sensitive and resistant NSCLC patients with EGFR activating exon 19 deletion mutations, therefore identifying those who could benefit from EGFR-TKIs and providing promising therapeutic opportunities.

In the present study, we identified three miRNAs potentially related with primary resistance to EGFR-TKIs in exon 19-mutated NSCLC. We subsequently utilized another cohort of 19 exon-mutated patients to validate this result. Moreover, we thought it is necessary to use another subgroup of patients with wild-type EGFR who had matched short and long PFS to verify reversely that the identified miRNAs in the first two groups contribute to primary resistance of EGFR 19 del NSCLC patients, but have no relation with resistance of EGFR wild-type NSCLC patients. Forty-eight EGFR wild-type patients came from our database; we intentionally chose this subgroup of patients with the matched longest and shortest PFS. Multiple studies have suggested that a small subgroup of patients without EGFR mutation (around $10 \%$ ) also can benefit from EGFRTKIs therapy; it might attribute to some uncommon mutations of EGFR (such as G719 and L861) [21] or other unknown mechanisms for now. What is more, in our database, we found that around $50 \%$ of patients in

Table 3 Validated significantly differentially expressed miRNAs in the plasma of responsive and resistance groups in 17 couple of patients with EGFR 19 mutation

\begin{tabular}{llll}
\hline miRNA & Responsive group & Resistant group & P-value \\
\hline miR-21 & $135.98 \pm 26.44$ & $182.91 \pm 47.63$ & 0.01 \\
miR-27a & $22.38 \pm 5.66$ & $131.85 \pm 53.75$ & 0.01 \\
miR-218 & $1.59 \pm 0.714$ & $5.09 \pm 1.65$ & 0.03 \\
\hline a'Wilcoxon test $(P<0.05)$ & &
\end{tabular}


Table 4 Clinical characteristics of the EGFR wild type group

\begin{tabular}{lll}
\hline & $\begin{array}{l}\text { Responsive } \\
\text { Group }\end{array}$ & $\begin{array}{l}\text { Resistance } \\
\text { Group }\end{array}$ \\
\hline $\begin{array}{ll}\text { Gender } \\
\text { Male }\end{array}$ & 6 & 13 \\
Female & 18 & 11 \\
Age & 62 & 58 \\
Median & & \\
Smoking & 6 & 9 \\
Yes & 18 & 15 \\
No & & 18 \\
Pathology & 21 & 6 \\
Ade & 3 & 7 \\
Non-ade & & 17 \\
Stage & 4 & 2.1 (0.3-3.5) \\
IIIB & 20 &
\end{tabular}

this study underwent EML4-ALK detection (FISH or VATANA), and no positive cases were found. As we know, EML4-ALK fusion and EGFR mutation are often exclusive for most patients, and incidence rate of overlapping EML4-ALK fusion and EGFR sensitive mutation was only about $0-8 \%$ in NSCLC patients $[5,22]$.
In our study with TaqMan miRNA array and qRT-PCR confirmation, 12 deregulated miRNAs were predicted by TargetScan in conjunction with bioinformatics methods (miR-448, miR-605, miR-628-5p, miR-561, miR-520f, miR-409-3p, has-miR-138, miR-296-5p, miR-218, miR1274B, miR-21, and miR-27a) in a training set. Three up-regulated miRNAs (miR-21, miR-27a, and miR-218) were identified in EGFR-mutant NSCLC patients resistant to EGFR-TKIs in the testing and validating cohorts.

High levels of miR-21 expression have been reported in various types of human tumors including lung cancer [23-25]. However, the mechanism that up-regulates miR-21 during carcinogenesis has not been identified yet. Seike et al. reported in 2009 that miRNA microarray data showed higher levels of miR-21 in EGFR-mutant cases [26], and in vitro analyses using NSCLC cell lines showed that activated EGFR signaling up-regulated miR21 expression. A statistically significant positive correlation was observed between miR-21 expression levels and p-EGFR levels in NSCLC cell lines. Furthermore, treatment with the EGFR-TKI (AG1478) inhibited miR21 expression in two NSCLC cell lines with elevated pEGFR, EGFR-mutant H3255, and EGFR wild-type H441, providing a mechanistic link between an activated EGFR signaling pathway and the aberrant up-regulation of miR-21, and a therapeutic basis for the inhibition of miR-21 in lung cancers with EGFR activation. Following antisense oligonucleotide-mediated knockdown of miR-

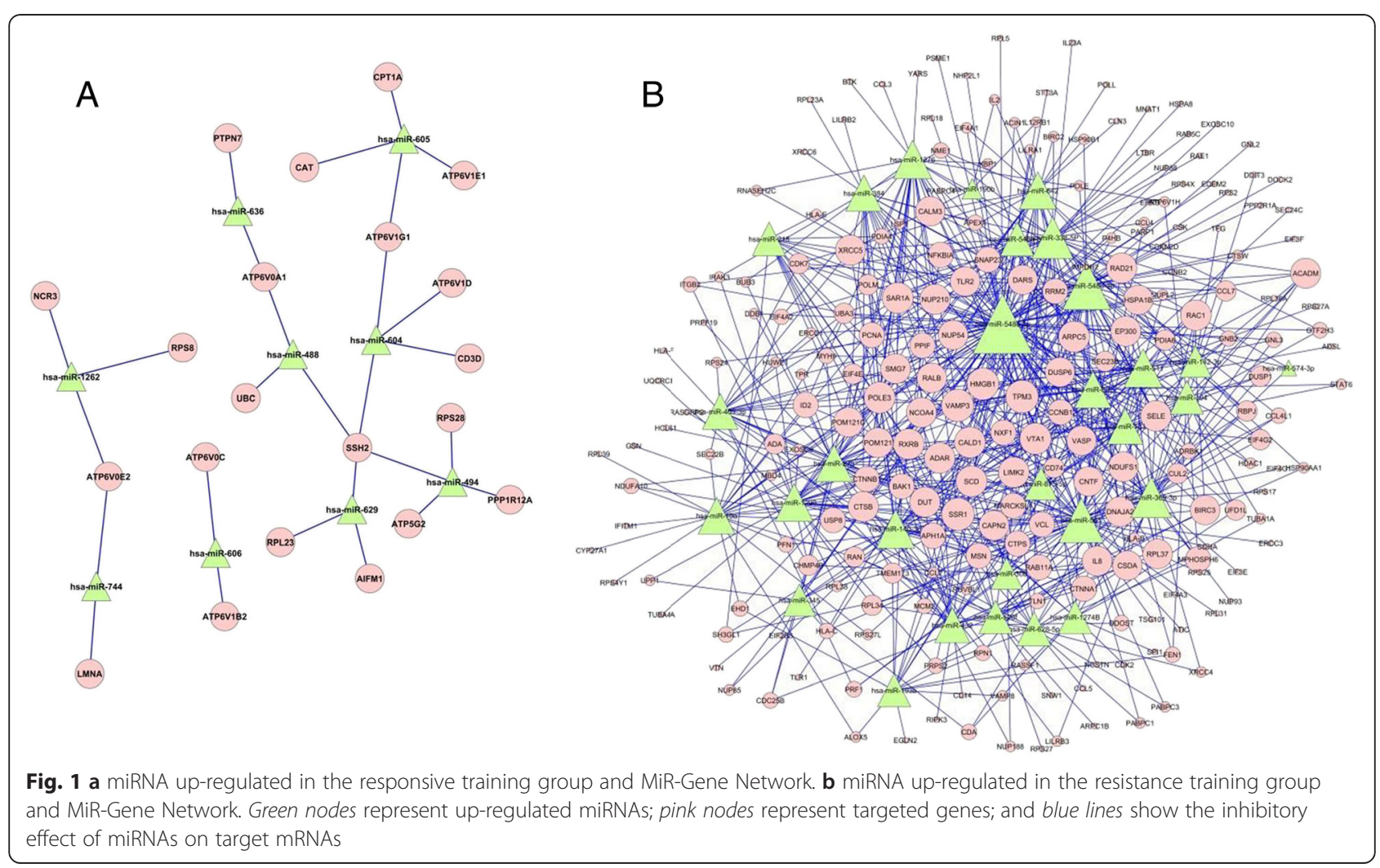


21, induced or enhanced apoptotic responses in two NSCLC cell lines, H3255 and H441, suggested that miR-21 could also be a therapeutic target in resistant EGFR-mutant NSCLC. Recent reports suggested that miR-21 overexpression was associated with resistance to cytotoxic agents, including gemcitabine [27], docetaxel [28], temozolomide [29], and taxan [30]. A recent study [31] based on animal model and clinical samples clarified that miR-21 overexpression was associated with acquired resistance to EGFRTKI in NSCLC, which might be caused by miR-21's function in activating the PI3K/AKT pathway through PTEN and PDCD4 inhibition. In our study, we identified miR-21 as a potential biomarker of resistance to EGFR-TKIs in EGFR-mutant patients. This is the first study that identified a potential association between miR-21 and primary resistance to EGFR-TKIs in EGFR-mutant patients. This finding needs to be further verified with in vitro and in vivo studies of molecular mechanisms and in larger clinical studies.

Studies involving associations of miR-27a and miR-218 with resistance to EGFR-TKIs in NSCLC are extremely limited for now. T790M mutation and MET gene amplification have been considered as important acquired resistant mechanisms. Recently, several studies also reported that T790M mutation or MET gene amplification could co-exist with EGFR sensitive mutation in therapynaïve patients $[20,32]$, suggesting that these two mechanisms may play important roles in primary resistance to EGFR-TKIs. Yoon and his colleagues [33] identified a mechanism linking miR-27a, MET, and EGFR that involves Sprouty2, and thus illustrating cross-talk between MET and EGFR in NSCLC. This study also showed that EGFR and MET receptor tyrosine kinases, through regulation of expression of specific miRNAs such as miR-218, control the metastatic behavior and gefitinib resistance of NSCLCs. Garofalo et al. [34] reported that the modulation of specific miRNAs, such as miR-30b, miR-30c, miR-221, and miR-222, might play a role in acquired resistance to EGFR-TKIs and could have therapeutic applications to sensitize lung tumors to TKI therapy [35]. However, these studies mainly focused on the association of plasma miRNA and MET in acquired resistance to EGFR-TKIs. The present study is the first to identify that miR-27a, miR-218, and miR-27a might play a role in primary resistance to EGFR-TKI in advanced NSCLC patients with EGFR exon 19 deletion mutations treated with EGFR-TKI, independent of c-MET amplification and T790M mutation. Other relevant mechanisms should be further investigated in basic research [36-38].

\section{Conclusion}

In summary, increased expression of miR-21, AmiR-27a, and miR-218 may play a role in primary resistance to
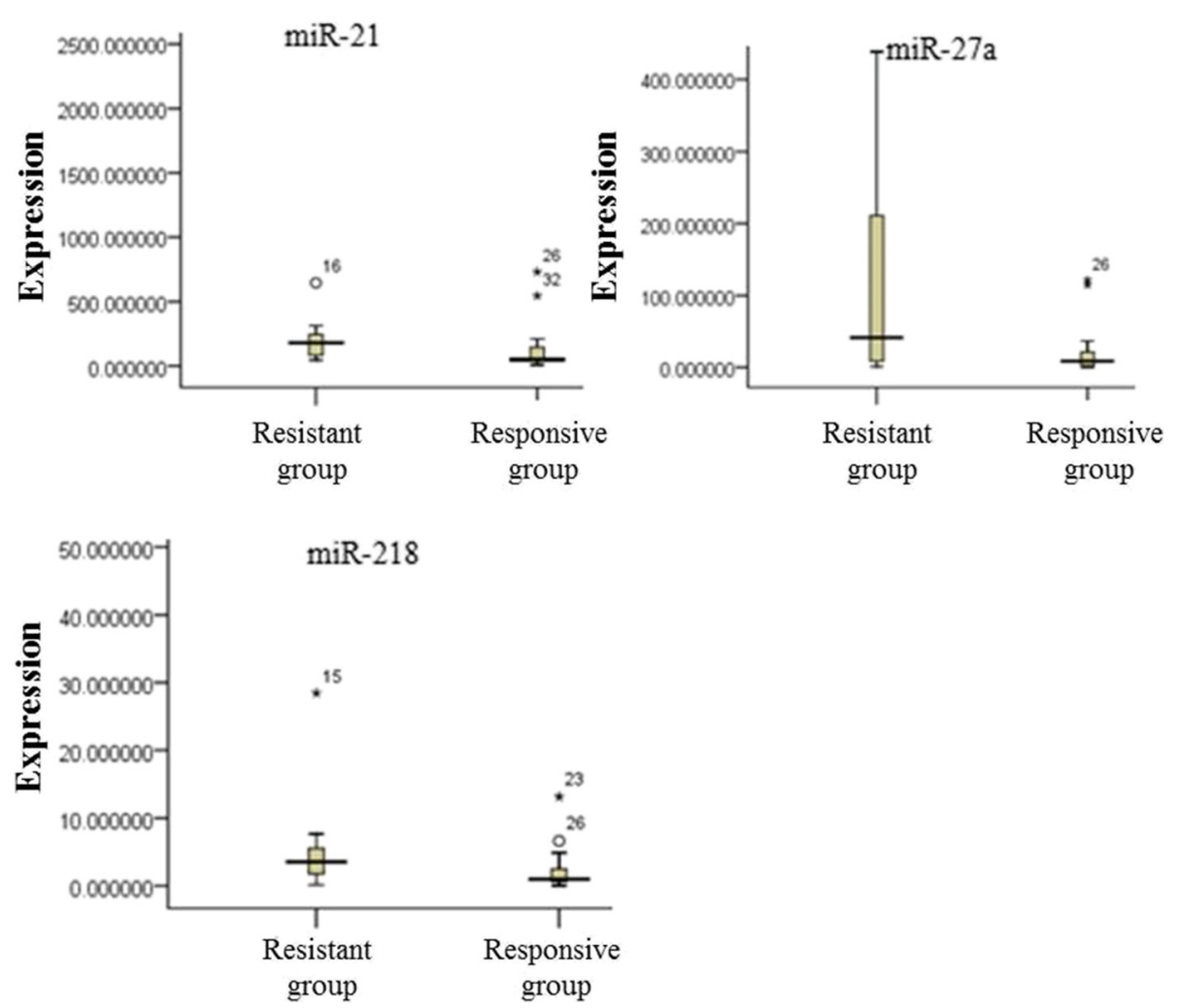

Fig. 2 Expression level of miR-21, miR-27a, and miR-218 in the plasma of the 34 patients of the validation group 
EGFR-TKI in advanced NSCLC patients who had EGFR exon 19 deletion mutations. This finding needs to be further confirmed in clinical study with a larger sample size. Finally, we propose that miRNAs targeted treatment combined with TKIs might provide a new strategy to treat NSCLCs in the future.

\begin{abstract}
Abbreviations
3'UTR: $3^{\prime}$ untranslated region; ARMS: amplification refractory mutation system; CR: complete response; DHPLC: denaturing high-performance liquid chromatography; EGFR-TKIs: epidermal growth factor receptor tyrosine kinase inhibitors; GO: gene ontology; IPA: ingenuity pathway analysis; KEGG: Kyoto encyclopedia of genes and genomes; NSCLC: non-small cell lung cancer; OS: overall survival; PDCD4: programmed cell death gene 4; PFS: progression-free survival; PR: partial response; qRT-PCR: quantitative reverse transcriptase PCR; RECIST: Response Evaluation Criteria in Solid Tumors; SD: stable disease; TLDA: TaqMan low-density array.
\end{abstract}

\section{Competing interests}

The author(s) declare that they have no competing interests.

\section{Authors' contributions}

SW disign the study, compose the manuscript. XS carry out research in lab, sumarize data, compose the manuscript. HB carry out research in lab, sumarize data. JZhao collect clinical sample and data. JD collect clinical sample and data. TA collect clinical sample and data. MZ collect clinical sample and data. ZW collect clinical sample and data. MW collect clinical sample and data. ZL edit the manuscript. JZhu edit the manuscript. JW disign the study, direct the research, compose the manuscript. All authors read and approved the final manuscript.

\section{Acknowledgement}

We gratefully acknowledge the valuable cooperation of Dr. Guoshuang Feng from Chinese Center for Disease Control and Prevention who made a great contribution to the statistics for our study.

This project was supported by the National Natural Science Foundation of China (Grant No. 81101726).

\section{Author details}

'The Key Laboratory of Carcinogenesis and Translational Research (Ministry of Education), Beijing, China. ${ }^{2}$ Department of Thoracic Medical Oncology, Peking University School of Oncology, Beijing Cancer Hospital and Institute, 100036 Beijing, China. ${ }^{3}$ Department of Bioscience and Nutrition, Novum, Karolinska Institute, 14183 Huddinge, Sweden.

Received: 25 May 2015 Accepted: 28 September 2015

Published online: 12 November 2015

\section{References}

1. Mok TS, Wu YL, Thongprasert S, Yang CH, Chu DT, Saijo N, et al. Gefitinib or carboplatin-paclitaxel in pulmonary adenocarcinoma. N Engl J Med. 2009;361(10):947-57.

2. Zhou C, Wu YL, Chen G, Feng J, Liu XQ, Wang C, et al. Erlotinib versus chemotherapy as first-line treatment for patients with advanced EGFR mutation-positive non-small-cell lung cancer (OPTIMAL, CTONG-0802): a multicentre, open-label, randomised, phase 3 study. Lancet Oncol. 2011;12(8):735-42

3. Nakagawa T, Takeuchi S, Yamada T, Ebi H, Sano T, Nanjo S, et al. EGFR-TKI resistance due to BIM polymorphism can be circumvented in combination with HDAC inhibition. Cancer Res. 2013;73(8):2428-34.

4. Pao W, Wang TY, Riely GJ, Miller VA, Pan Q, Ladanyi M, et al. KRAS mutations and primary resistance of lung adenocarcinomas to gefitinib or erlotinib. PLoS Med. 2005;2(1), e17.

5. Shaw AT, Yeap BY, Mino-Kenudson M, Digumarthy SR, Costa DB, Heist RS, et al. Clinical features and outcome of patients with non-small-cell lung cancer who harbor EML4-ALK. J Clin Oncol. 2009;27:4247-53.

6. Kumar MS, Lu J, Mercer KL, Golub TR, Jacks T. Impaired microRNA processing enhances cellular transformation and tumorigenesis. Nat Genet. 2007;39:673-7.

7. Eder M, Scherr M. MicroRNA and lung cancer. N Engl J Med. 2005;352:2446-8.
8. Bianchi F, Nicassio F, Marzi M, Belloni E, Dall'olio V, Bernard L, et al. A serum circulating miRNA diagnostic test to identify asymptomatic high-risk individuals with early stage lung cancer. EMBO molecular medicine. 2011;3:495-503.

9. Yuan X, Wu H, Han N, Xu H, Chu Q, Yu S, et al. Notch signaling and EMT in non-small cell lung cancer: biological significance and therapeutic application. Journal of Hematology Oncology. 2014;7(1):87.

10. Ahmad A, Maitah MY, Ginnebaugh KR, Li Y, Bao B, Gadgeel SM, et al. Inhibition of hedgehog signaling sensitizes NSCLC cells to standard therapies through modulation of EMT-regulating miRNAs. J Hematol Oncol. 2013;6:77.

11. Hennessey PT, Sanford T, Choudhary A, Mydlarz WW, Brown D, Adai AT, et al. Serum microRNA biomarkers for detection of non-small cell lung cancer. PLoS One. 2012;7, e32307.

12. Boeri M, Verri C, Conte D, Roz L, Modena P, Facchinetti F, et al. MicroRNA signatures in tissues and plasma predict development and prognosis of computed tomography detected lung cancer. Proc Natl Acad Sci U S A. 2011;108:3713-8.

13. Shen J, Todd NW, Zhang H, Yu L, Lingxiao X, Mei $Y$, et al. Plasma microRNAs as potential biomarkers for non-small-cell lung cancer. Laboratory investigation; a journal of technical methods and pathology. 2011;91:579-87.

14. Wang $W-T$, Chen $Y-Q$. Circulating miRNAs in cancer: from detection to therapy. J Hematol Oncol. 2014;7(1):86.

15. Smith AD, Roda D, Yap TA. Strategies for modern biomarker and drug development in oncology. Journal of Hematology Oncology. 2014;7:70.

16. Lee JK et al. Primary resistance to epidermal growth factor receptor (EGFR) tyrosine kinase inhibitors (TKIs) in patients with non-small-cell lung cancer harboring TKI-sensitive EGFR mutations: an exploratory study. Ann Oncol. 2013;24(8):2080-7.

17. Zhang Y, Sheng J, Kang S, et al. Patients with exon 19 deletion were associated with longer progression-free survival compared to those with L858R mutation after first-line EGFR-TKls for advanced non-small cell lung cancer: a meta-analysis. PLoS One. 2014;9(9), e107161.

18. Eisenhauer EA, Therasse P, Bogaerts J, Schwartz LH, Sargent D, Ford R, et al New response evaluation criteria in solid tumours: revised RECIST guideline (version 1.1). European Journal Of Cancer. 2009;45:228-447.

19. Bai H, Mao L, Wang HS, Zhao J, Yang L, An TT. Epidermal growth factor receptor mutations in plasma DNA samples predict tumor response in Chinese patientswith stages IIIB to IV non-small-cell lung cancer. J Clin Oncol. 2009;27(16):2653-9.

20. Wang Z, Chen R, Wang S, Zhong J, Wu M, Zhao J, et al. Quantification and dynamic monitoring of EGFR T790M in plasma cell-free DNA by digital PCR for prognosis of EGFR-TKI treatment in advanced NSCLC. PLOS One. 2014:9(11), e110780.

21. Wu J-Y, Yu C-J, Chang Y-C, et al. Effectiveness of tyrosine kinase inhibitors on "uncommon" epidermal growth factor receptor mutations of unknown clinical significance in non-small cell lung cancer. J Thorac Oncol. 2014;9(2):189-94.

22. Zhang S, Wang F, Keats J. Crizotinib-resistant mutants of EML4-ALK identified through an accelerated mutagenesis screen. Chem Biol Drug Des. 2011;78(6):999-1005.

23. Meng F, Henson R, Wehbe-Janek H, Ghoshal K, Jacob ST, Patel T, et al. MicroRNA-21 regulates expression of the PTEN tumor suppressor gene in human hepatocellular cancer. Gastroenterology. 2007;133:647-58.

24. Chan JA, Krichevsky AM, Kosik KS. MicroRNA-21 is an antiapoptotic factor in human glioblastoma cells. Cancer Res. 2005;65:6029-33.

25. Yanaihara N, Caplen N, Bowman E, Seike M, Kumamoto K, Yi M, et al. Unique microRNA molecular profiles in lung cancer diagnosis and prognosis. Cancer Cell. 2006;9:189-98.

26. Seike M, Goto A, Okano T, Bowman ED, Schetter AJ, Horikawa I, et al. MiR-21 is an EGFR-regulated anti-apoptotic factor in lung cancer in never-smokers. Proc Natl Acad Sci U S A. 2009;106(29):12085-90.

27. Giovannetti E, Funel N, Peters GJ, Del Chiaro M, Erozenci LA, Vasile E, et al. MicroRNA-21 in pancreatic cancer: correlation with clinical outcome and pharmacologic aspects underlying its role in the modulation of gemcitabine activity. Cancer Res. 2010;70:4528-38.

28. Shi GH, Ye DW, Yao XD, Zhang SL, Dai B, Zhang HL, et al. Involvement of microRNA-21 in mediating chemo-resistance to docetaxel in androgen-independent prostate cancer PC3 cells. Acta Pharmacol Sin. 2010;31:867-73.

29. Shi L, Chen J, Yang J, Pan T, Zhang S, Wang Z, et al. MiR-21 protected human glioblastoma U87MG cells from chemotherapeutic drug 
temozolomide induced apoptosis by decreasing $\mathrm{Bax} / \mathrm{BCl}-2$ ratio and caspase-3 activity. Brain Res. 2010;1352:255-64.

30. Ren Y, Zhou X, Mei M, Yuan XB, Han L, Wang GX, et al. MicroRNA-21 inhibitor sensitizes human glioblastoma cells U251 (PTEN-mutant) and LN229 (PTEN-wild type) to taxol. BMC Cancer. 2010;10:27.

31. Li B, Ren S, Li X, Wang Y, Garfield D, Zhou S, et al. MiR-21 overexpression is associated with acquired resistance of EGFR-TKI in non-small cell lung cancer. Lung Cancer. 2014;83(2):146-53.

32. Cross DA, Ashton SE, Ghiorghiu S, Eberlein C, Nebhan CA, Spitzler PJ, et al. AZD9291, an irreversible EGFR TKI, overcomes T790M-mediated resistance to EGFR inhibitors in lung cancer. Cancer Discov. 2014;4(9):1046-61.

33. Yoon KA, Yoon H, Park S, Jang HJ, Zo Jl, Lee HS, et al. The prognostic impact of microRNA sequence polymorphisms on the recurrence of patients with completely resected non-small cell lung cancer. J Thorac Cardiovasc Surg. 2012;144(4):794-807.

34. Garofalo M, Di Leva G, Romano G, Nuovo G, Suh SS, Ngankeu A, et al. MiR-221\&222 regulate TRAll resistance and enhance tumorigenicity through PTEN and TIMP3 downregulation. Cancer Cell. 2009;16:498-509.

35. Niu F-Y, Wu Y-L. Novel agents and strategies for overcoming EGFR TKIs resistance. Exp Hematol Oncol. 2014;3(1):2

36. Zhang H, Chen Z, Wang X, Huang Z, He Z, Chen Y. Long non-coding RNA: a new player in cancer. J Hematol Oncol. 2013;6:37.

37. Zhuang $Y$, Wang $X$, Nguyen HT, Zhuo $Y$, Cui $X$, Fewell $C$, et al. Induction of long intergenic non-coding RNA HOTAIR in lung cancer cells by type collagen. J Hematol Oncol. 2013;6:35.

38. Loewen $G$, Jayawickramarajah J, Zhuo Y, Shan B. Functions of IncRNA HOTAIR in lung cancer. J Hematol Oncol. 2014;7(1):90.

\section{Submit your next manuscript to BioMed Central and take full advantage of:}

- Convenient online submission

- Thorough peer review

- No space constraints or color figure charges

- Immediate publication on acceptance

- Inclusion in PubMed, CAS, Scopus and Google Scholar

- Research which is freely available for redistribution 